\title{
TWO WAYS OF IMMEDIATE REHABILITATION OF EDENTULOUS MANDIBLE WITH DENTAL IMPLANTS AND PROSTHESES - CRITICAL VIEW ON BRÅNEMARK SYSTEM ${ }^{\circledR}$ NOVUM
}

\begin{abstract}
Jiři Krug, Radek Mounajjed
D.C.M., private dental office, Hradec Králové

Summary: With appropriate stabilization and occlusal loading, mandibular implants can be immediately loaded in a complete-arch configuration with no apparent detrimental effect on the rates of osseointegration. Ten patients were treated with the immediate loading protocols in completely edentulous mandible either with conventional one-stage implants and a provisional denture or with the Brånemark System ${ }^{\circledR}$ Novum and a definite restoration. A total of 42 implants were placed. One Novum implant was lost 1 month after placement. The clinical success rate of the immediately loaded implants at the time of the 6-month check-up was $97.6 \%$. Radiographic surveys completed by panoramic radiographs 6 months after the surgery revealed good healing of bone at implant interface. After the period, mean marginal bone loss was 0.4 $\mathrm{mm}$ (ranged from $0 \mathrm{~mm}$ to $1.5 \mathrm{~mm}$ ). The results of this short-term clinical and radiographic study indicate that the precise surgical and prosthetic protocol allows successful prosthetic rehabilitation of mandibular edentulism and that the provisional or permanent fixed reconstruction can be provided to a patient on a day of fixture surgery. Finally, authors consider critical comments on Brånemark System ${ }^{\circledR}$ Novum.
\end{abstract}

Key words: Dental implants; Fixed prosthesis; Immediate loading; Surgical techniques; One-stage method; Edentulous

\section{Introduction}

Oral rehabilitation with endosseous implant-supported restorations has become an accepted treatment modality because of the high level of predictability that can be achieved using this methodology (3). Based on the original work of Adell and co-workers (1) and Brånemark et al. (5), clinicians long believed that a period of stress-free healing after implant placement was an essential requirement for osseointegration. These and other authors (6) concluded that motion between an endosseous screw and bone resulted in connective tissue proliferation and fibrous encapsulation of the screw. Most of the endosseous cylindric dental implant systems developed recommended a 3- to 6-month unloaded healing period for successful osseointegration (15). An exception to this protocol was the titanium plasma-sprayed (TPS) screw developed by Straumann AG (Waldenburg, Switzerland), which was designed to be loaded using a cast bar and overdenture immediately following surgical placement in the mandible (7). In fact, the length of the healing period for the machined screw-type implants was countered on the empirical follow-up, not on experimental studies. Currently, important factors like bone quality, technique of implant placement, splinting of fixtures, design and surface of implants are primarily counted when making decision on healing period. There is a question, whether the unloaded phase is necessary for complete osseointegration or if under certain condition one may load fixtures earlier not jeopardizing the survival of implants. It is necessary to note that even during the healing phase especially the one-stage implants are always under certain loading. Pilliar and co-workers (22) reported that micromovement reaching $150 \mu \mathrm{m}$ can disturb fate of osseointegration; on the other hand even repeatable movement ranging in $50 \mu \mathrm{m}$ is well tolerated. The critical level of the movement is somewhere between 50 and $150 \mu \mathrm{m}$ and depends on implant design and its surface.

Several articles were published with clinical evaluation of immediate or early loaded implants. Schnitman et al. (24) examined the use of immediately loaded Brånemark implants for the support of temporary fixed bridges in the interforaminal area in the mandible, and published the results of a follow-up study that was undertaken ten years later. The first results were promising but later, implant survival was reduced in comparison to standard methods. Chiapasco and associated (8) documented 5-year implant success rates of $97 \%$. Chow et al. (9) reported a prospective clinical study evaluating immediate loading of four Brånemark fixtures in the mandible. The survival rate of $100 \%$ was seen after 1 year in function. The mean marginal bone loss amounted to $0.64 \mathrm{~mm}$. Randow et al. (23) ma- 
naged 16 patients with edentulous lower jaw by means of 88 dental implants. Fixed prostheses were delivered within 20 days after surgery. They compared the marginal bone loss between these patients and others where two-phase implants were conventionally loaded 4 months after their placement. Eighteen months later, a mean bone loss was in immediate loaded implants lower $(0.4 \mathrm{~mm})$ than in control group $(0.8 \mathrm{~mm})$. There has not been reported significant difference between immediate, early or delayed loaded implants considering the quality of osseointegration, no fibrous capsule was found around implants $(8,23,24)$. Interesting study published Piatelli and co-workers (21), who histomorphometrically examined bone blocks with dental implants placed in jaws of monkeys that were immediately splinted together with crowns, and as well loaded. After 9 months, he noticed layers of lamellar bone adjacent to implant fixture that were significantly thicker than around non loaded implants. Splinting of implants together is an effective method how to reduce mechanical overloading during their early function.

With the purpose of immediate loading, we can use any implant system with one-stage fixtures. The implants are connected either with provisional or permanent abutments during the surgery, before soft tissue is sutured. The current trend of immediate implant placement and especially immediate implant loading has encouraged implant companies to produce appropriate screw implants for this purpose. Even the producers, which are well known for their twophase implants, offer fixtures suitable for immediate loading. Brånemark System ${ }^{\circledR}$ Novum (Nobel Biocare, Gothenburg, Sweden) was introduced in 1999 as an immediate loading alternative to the conventional mandibular hybrid restoration (fixed denture) for edentulous patients (4). It consists of 3 one-stage implants placed precisely between the mental foraminae that immediately support (at the same day) a definitive fixed prosthesis fabricated on premachined components. This technology has been used in Sweden for over 5 years with very high prosthesis and implant success rates of $98 \%$ (11). More importantly, the rates of crestal bone loss around the supportive implants are shown by the same investigators to be essentially the same as those for the conventional delayed approach, which endorses both the efficacy and safety of this biologic response to immediate loading (4). The most challenging aspect of the Novum concept is the surgical technique once the patient selection process has been successfully completed. Nevertheless, different techniques are presented as a surgical bone reduction guide or an implant placement guide (20), performing Novum System demands advanced professionals.

\section{Materials and methods}

\section{Patient Selection and Preoperative Planning}

Ten patients ( 6 men and 4 women) with an age range of 48 to 65 years and a mean age of 58 years were referred to our private dental office for treatment of their mandibular arches. All patients were either completely edentulous or were soon to be edentulous because of advanced caries or periodontal disease and desired fixed restoration. Their health was good or non-contributory; two patients smoked 20 cigarettes per day, 1 patient had controlled Type II diabetes, and 4 had controlled hypertension. In the opposing maxillary arches, 7 patients wore removable prostheses and 3 had fixed restorations. In 2 patients, it was determined that the maxillary plane was not conducive to establish the occlusal goals, and fabrication of a new denture prior to the treatment of the mandibular arch was made. After consulting with the patients, the radiographs were evaluated to ensure that there was adequate bone height available for placement of five to six implants between the mental foraminae. We indicated the Brånemark System ${ }^{\circledR}$ Novum for patients with height of the mandible lower than $15 \mathrm{~mm}$ in interforaminal region. Others were planned for a conventional hybrid denture. After implant placement, the patients were followed-up for 6 months and checked clinically and radiographically. Panoramic radiographs were taken at the time of delivery of a restoration and 6 months later. Marginal bone loss was measured on panoramic radiographs. Postoperative follow-up of all ten patients ranged from 6 to 14 months, with a mean of 9.8 months.

\section{Surgical and prosthetic techniques}

Before the surgery, all patients were premedicated with oral antibiotics. Local anesthesia was obtained via bilateral inferior alveolar nerve blocks and local infiltration. Fullthickness crestal incision was made. The remaining teeth were extracted and socket thoroughly degranulated and curetted. In five patients, 5-6 conventional one-stage implants were placed in the mandible. The number of fixtures depended on the quality of restoration in the opposite jaw, mastication efficiency of a patient or existing parahabitual movement of the mandible. The strategy in implant positioning was to place them as parallel as possible in symphysal and parasymphysal area. Since most of the patients with edentulous lower jaw had resorption of the alveolar crest, we did not place implants laterally from the mental foramen. For the conventional treatment, we used standard solid implants ITI Straumann AG (Waldenburg, Switzerland) with SLA surface (sand blasted, large grit, and acid etched). Bone tapping was used in all implants. Prosthetic provisional abutments for ITI implants were tightened to the stable implants and interrupted sutures were placed interproximally to loosely adapt the flaps to the implants (Fig. 1). In this type of reconstruction, we adjusted already existing conventional denture. A curvilinear hole in the body of this restoration was made at the abutment sites. Self-curing not heat producing resin was used to fill the spaces between the abutments and the denture. We used a rubber dam to isolate the fixtures from the polymerization process and to shield the suture line and tissue from the acrylic resin. When acrylic material was set, the posterior saddles and denture flanges were reduced and the 


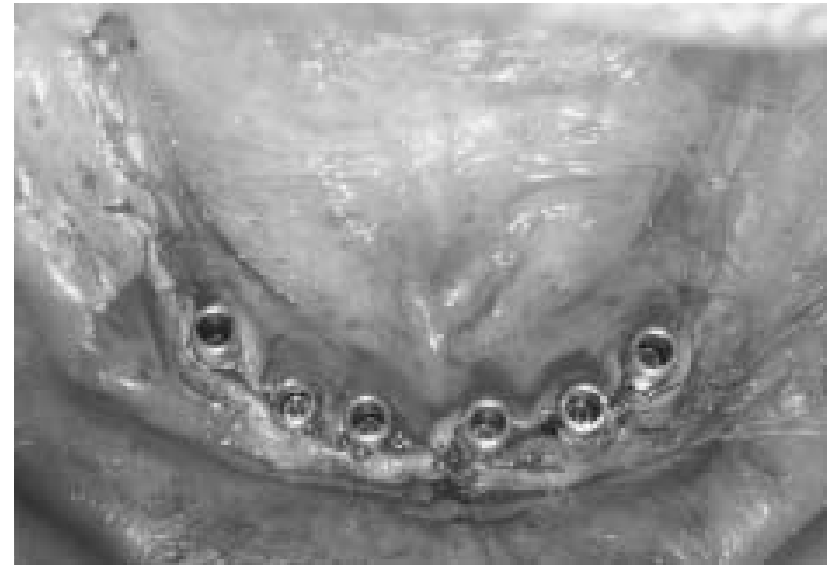

Fig. 1: Patient with 6 ITI implants placed in the edentulous lower jaw and connected with prosthetic provisional abutments immediately after surgery. Soft tissue was closed with interrupted sutures.

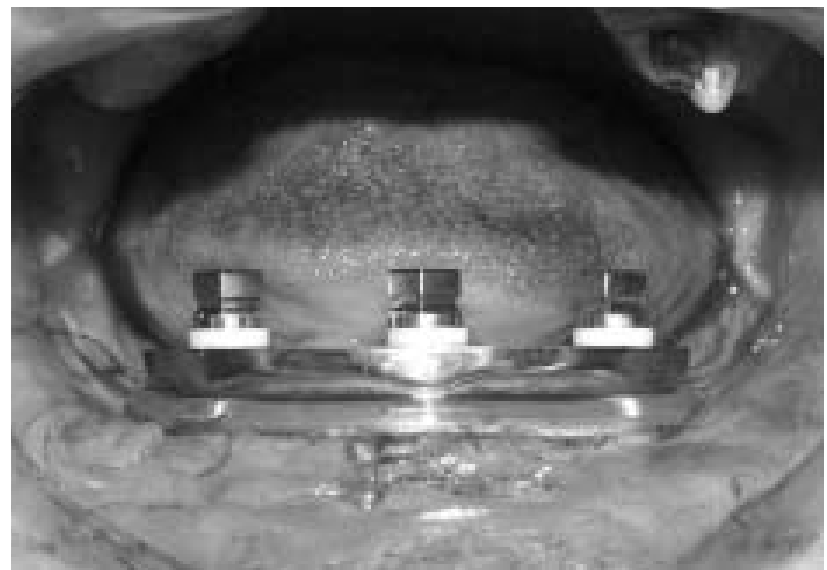

Fig. 3: Three parallelly placed implants connected with the lower bar. Sutures were protected under the interpositioned polyethylen plate.

denture became a provisional full implant-bearing restoration (Fig. 2). The relined denture was trimmed to provide access for cleaning, and the occlusion was verified to provide bilaterally equivalent contacts in centric relation and to provide balance, if occluding a new complete denture. All patients were asked to limit their diet to liquids for the first week following therapy. During the second and third weeks, patients were asked to avoid any hard foods requiring excessive tearing or incising in their diet. Following the forth week, patients were unrestricted in their diet. Hygiene was reinforced and a water irrigation device was recommended along with proximal brushes and superfloss. After 8 weeks, provisional abutments were replaced for final synOcta ${ }^{\circledR}$ abutments and definite hybrid denture was made and screwed to the implants.

Alternatively, we used the Brånemark System ${ }^{\circledR}$ Novum. According to the protocol, 3 implants were parallelly plac-

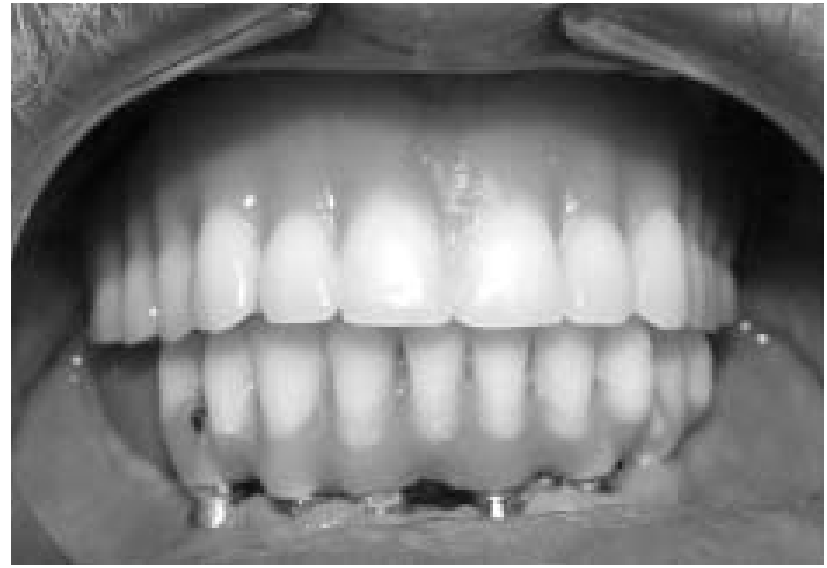

Fig. 2: Provisional full implant-bearing restoration cemented to the abutments.

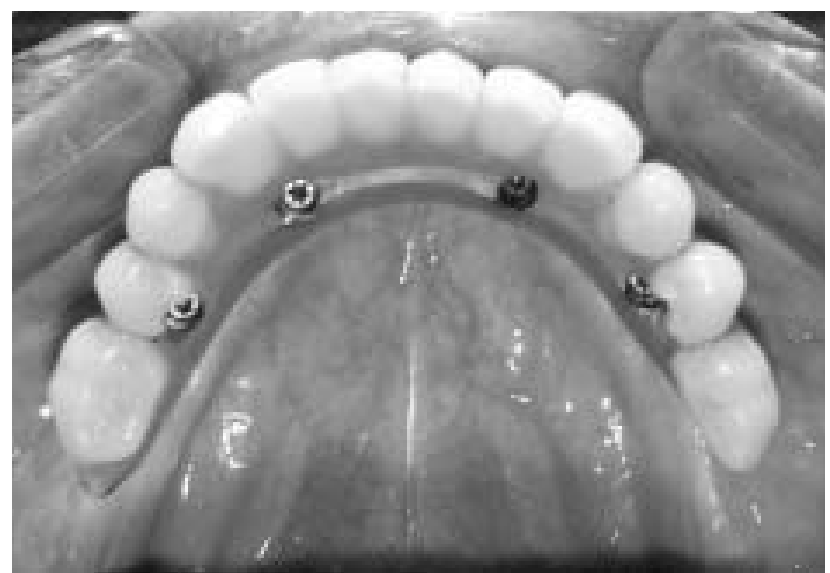

Fig. 4: Procedure was finished in a conventional way and the upper bar together with the acrylic denture was connected to the lower bar by means of 4 screws.

ed in the symphysis in the pretapped beds of the lower jaw. Before definite connection with the lower bar, polyethylen plate was interpositioned and soft tissue was closed with interrupted sutures (Fig. 3). Temporarily, the upper bar was fixed to the lower bar and the jaw relation reconstruction was performed. To complete the reconstruction, we used two techniques; either using utility red wax or silicon material of very heavy consistency (putty) adhered to the upper bar. The relation was transferred to an articulator and teeth were set in the wax followed by trial of the denture in a patient's mouth. We finished the procedure in a conventional way and the upper bar together with the acrylic denture was connected by means of 4 screws to the lower bar (Fig. 4). The acrylic teeth were adjusted according to the long centric relation and to provide balance. One week after, we removed stitches and the silicon plate, and tightened the screws if necessary. 


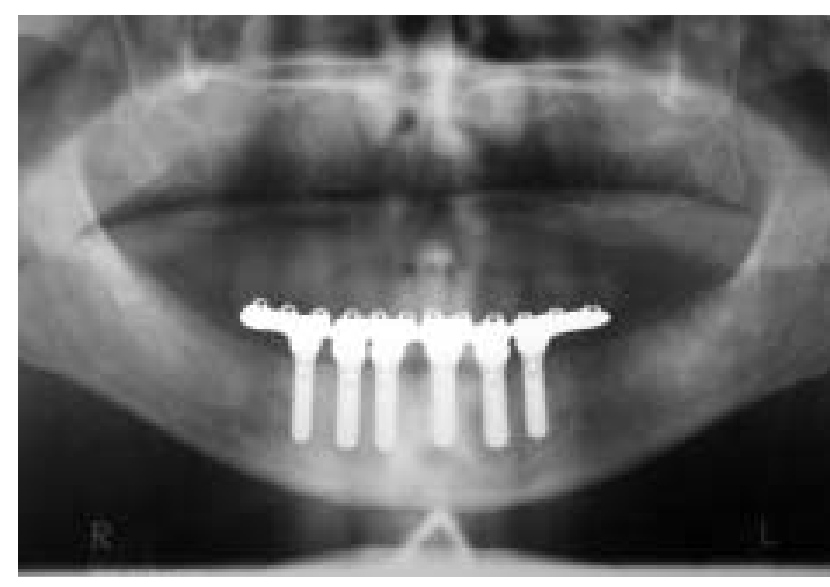

Fig. 5: Panoramic radiograph with 6 ITI implants placed in the lower jaw 8 months after surgery.

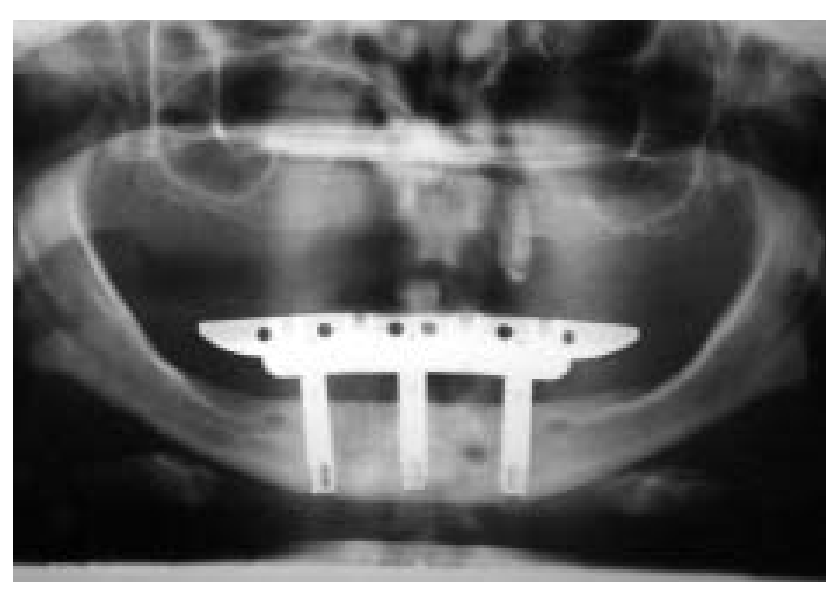

Fig. 6: Panoramic radiograph with 3 Brånemark System ${ }^{\circledR}$ Novum implants placed in the lower jaw 6 months after surgery.

Tab: 1: Summary of implants placed, restoration types, and patients data.

\begin{tabular}{|c|c|c|c|c|c|c|c|}
\hline patient & $\begin{array}{c}\text { Implant } \\
\text { type/surface }\end{array}$ & $\begin{array}{c}\text { No/length/O } \\
\text { of implants }\end{array}$ & $\begin{array}{c}\text { Opposing } \\
\text { occlusion }\end{array}$ & $\begin{array}{c}\text { Restoration } \\
\text { type }\end{array}$ & $\begin{array}{c}\text { No. of failed } \\
\text { implants }\end{array}$ & $\begin{array}{c}\text { Age (y) at } \\
\text { placement }\end{array}$ & $\begin{array}{c}\text { Months } \\
\text { loaded }\end{array}$ \\
\hline J.F. & ITI, SLA & $6 / 14 / 4.1$ & removable & LPC & 0 & 62 & 16 \\
\hline J.B. & ITI/SLA & $5 / 14 / 4.1$ & removable & OPC & 0 & 65 & 10 \\
\hline S.S. & ITI/SLA & $6 / 14 / 4.1$ & fixed & LPC & 0 & 48 & 9 \\
\hline I.D. & ITI/SLA & $5 / 14 / 4.1$ & removable & OPC & 0 & 51 & 6 \\
\hline F.L. & ITI/SLA & $5 / 14 / 4.1$ & removable & OPC & 0 & 64 & 6 \\
\hline C.B. & Novum/Mach. & $3 / 13.5 / 5.0$ & fixed & LPS & 1 & 52 & 14 \\
\hline J.P. & Novum/Mach. & $3 / 13.5 / 5.0$ & removable & LPS & 0 & 55 & 12 \\
\hline N.V. & Novum/Mach. & $3 / 13.5 / 5.0$ & removable & LPS & 0 & 56 & 11 \\
\hline V.K. & Novum/Mach. & $3 / 13.5 / 5.0$ & fixed & LPS & 0 & 62 & 8 \\
\hline M.ä. & Novum/Mach. & $3 / 13.5 / 5.0$ & removable & LPS & 0 & 51 & 6 \\
\hline
\end{tabular}

SLA = sand blasted, large grit, and acid etched; Novum = Brånemark System ${ }^{\circledR}$ Novum; Mach. $=$ machined; LPC $=$ laboratory-processed cemented; OPC = office-processed cemented; LPS = laboratory-processed screw-retained.

\section{Results}

Patient data are shown in Table 1. Ten patients were treated with the immediate loading protocols in completely edentulous mandible either with conventional one-stage implants and the provisional denture or with the Novum System and the definite restoration. A total of 42 implants were placed in 10 patients. All of them were deemed stable at the time of implant surgery and immediately loaded. One Novum implant was lost 1 month after placement with pain and mobility. This lateral implant was originally placed in two steps because of our technical mistake during preparing the bed. After explantation, the denture and the bars were removed. The failed implant was successfully replaced 3 months later and immediately loaded. After 8 months, during the final recall, that implant was firmly fixed. The clinical success rate of the immediately loaded implants at the time of the 6-month check-up was $97.6 \%$ (41 of 42 implants). The radiographic surveys completed by panoramic radiographs 6 months after the surgery revealed good healing of bone at implant interface (Fig. 5, 6). The cortical bone levels appeared to be maintained at the rough surface border or above the last superior thread of the machined fixture, respectively. A mean marginal bone loss was 0.4 $\mathrm{mm}$ (ranged from $0 \mathrm{~mm}$ to $1.5 \mathrm{~mm}$ ) during the measurement 6 months after implant placement.

\section{Discussion}

All patients presented with edentulous lower jaw were managed with dental implants and either with provisional cemented or fixed-removable restorations. It supported the statement that controlled immediate loading of one-stage implants placed in high dense bone (especially in symphyseal area of the lower jaw) did not disturb the process of osseointegration (13). The obtained results showed optimal clinical and radiographic integration of the implants 6 months postoperatively. Only 1 implant out of 42 failed, 
possibly due to the technical mistake that we made during implant placement. The implant was placed 7 months after teeth extraction in healthy bone. Otherwise, all implants were stable and without signs of inflammation during the following period. The decision for the Novum system was made on the height of the mandible only, if other anatomical propositions did not contraindicate the procedure. Regarding the jaw volume available for implants, jaws can be classified into 5 different shape groups, according to Lekholm \& Zarb (16). Of these, classes B, C, and D are appropriate for the Novum protocol, whereas class A should be treated with caution because the implants might not reach the second cortex. Class E is inappropriate, as there is too little bone for the Novum protocol fixtures (17).

It is proposed that a term of immediate loading should refer to situations where implant placement with primary stability and prosthetic loading usually with a provisional denture occur at the same clinical visit. Immediate loading of conventional implants must follow some principles to avoid early failures, since they could be the results of different procedural mistakes. Same-day loading is temporally irrelevant with respect to osseointegration (10). Biologically, primary stability of implants is a crucial requirement for the success. Bicortical anchorage must be maximized, whenever it is possible. The fixtures should be distributed in the widest arch possible within the anatomic limitation. Immediate loading of implants eliminates possible disruption of the blood clot that might appear during manipulation with a denture in the important early stages of healing. We should avoid removal of the provisionals within the time of osseointegration and provisional fabrication on early loading protocol. Rigid splinting increases the stability of the implants and together with minimal lateral force application are critical factors for the success. The forces exerted on the implants through fixed provisional restorations can apparently be minimized or reduced below the range of "deleterious micromovement". However, the patients must follow the diet regime and not to abuse the implants.

Number of implants used in our patients for the conventional hybrid denture appeared to be adequate to allow for clinical osseointegration and both provisional and definitive prosthesis fabrication. There does not appear to be a clear indication of the minimum implant support required. Empirically, the number of implants varied according to certain patient parameters, including the opposing dentition, mastication efficiency, and history of parafunctional habits. Chow et al. (9) experienced good results using 4 implants immediately loaded with a fixed denture. We used either 5 or 6 conventional one-stage implants. Alternatively, the Novum system counts 3 implants inter foraminae of the lower jaw. All implants used in this report were screw-type design either with rough surface (ITI SLA) or machined (Novum system). In humans, initial reports for early loading suggest that abutment connection and function 6 to 8 weeks after placement in types I, II, and III bone (according to Misch's classification) (18) is possible and predic- table (7). This reduced time for clinical osseointegration or "early loading" should be distinguished from immediate loading. Patients appeared to benefit from immediately loaded implant restorations in several tangible ways. They resumed function quickly following surgery and provisional restoration placement. Masticatory function is uniformly judged to be superior to pretreatment comparisons (10). Removable dentures can often inadvertently apply excessive forces, whether or not the implants are submerged below the flaps. The use of a fixed provisional restoration can help control the occlusal forces that are applied to the healing boneto-implant interface within a physiologic range (2). It has been suggested that several requirements should be met for clinical success with immediately loaded implants (Table 2).

Tab. 2: Factors influencing implants' resistance to movement.

\begin{tabular}{|l|}
\hline adequate number, length, and diameter of implants \\
\hline stabilizing characteristics of implants \\
\hline bone quality or density \\
\hline adequate distribution of implants \\
\hline good implant stabilization \\
\hline rigid provisional splinting \\
\hline physiologic occlusal scheme (12) \\
\hline
\end{tabular}

According to our observation of the patients and comments in reviewed literature, we express the following critical view on the Novum system:

1. Generally, the Novum system demands higher experience and advanced cooperation of all contributing persons - surgeon, prosthodontist, and technician. Only stabile, well oriented, and parallelly placed implants that are horizontally positioned at the same level can be followed for the immediate prosthetic loading.

2. For the successful results, we demand cooperative patients without limited mouth opening. The surgical part lasts 3 hours and total time of the treatment takes about 8 hours.

3. Each patient must have enough bone volume to allow a superior table of at least $6 \mathrm{~mm}$ in width to be created while leaving enough osseous depth for implant placement. Patients with local insufficient offer of the alveolar bone of the frontal area of the lower jaw (less than $6 \mathrm{~mm}$ vestibulo-orally and less $11 \mathrm{~mm}$ vertically) are contraindicated for the procedure. So far, the Novum system uses only two diameters of fixture $(4.5 \mathrm{~mm}$ and $5.0 \mathrm{~mm})$ and two lengths $(13.5 / 7 \mathrm{~mm}$ and $11.5 / 5 \mathrm{~mm})$. Since the Novum system is presently available in one dimension only, the patients with very narrow (V-shaped) mandible cannot be treated with the prefabricated bar. In cases where the alveolar crest is lower or narrower, augmentation should be used at first and then conventional implantation is proceeded. Moreover, in severely resorbed mandible, very short implants can be placed and implant supported overdenture can be worked-out. 


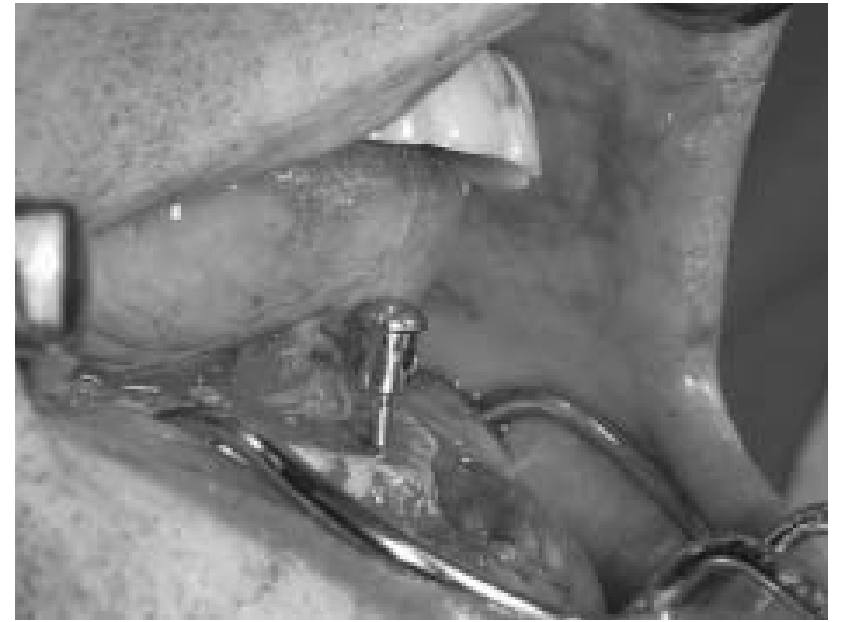

Fig. 7: Position of the first medial implant defines the final settling of the bar and more less the denture as well.

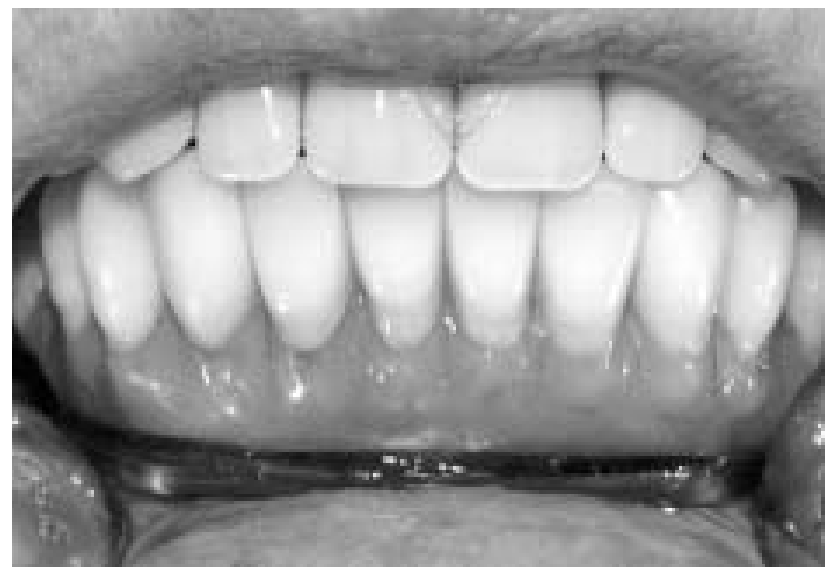

Fig. 8: For patients who desire advanced cosmetics, it is possible to cover the frontal rim of the lower bar with an acrylic veneer connected to the upper bar.

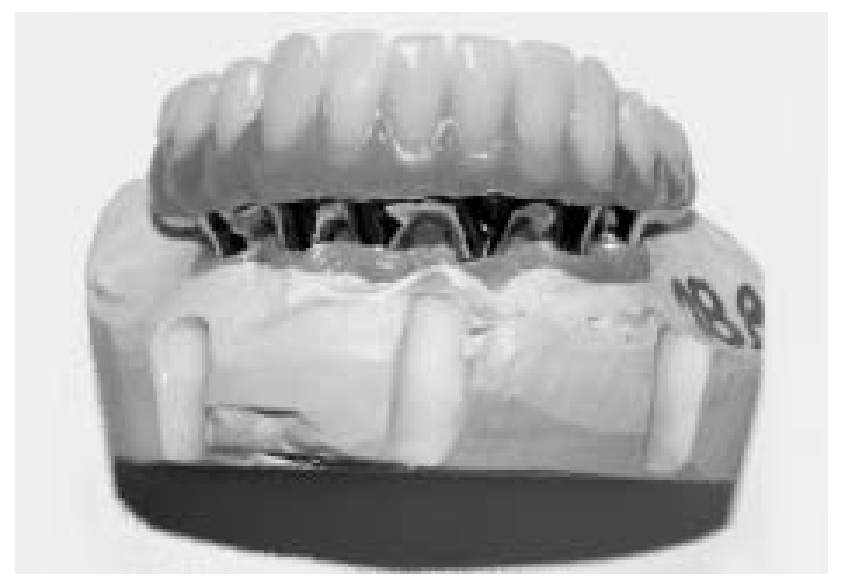

Fig. 9: Final restoration with more physiologically looking trimming is made on the conventional protocol.
4. To avoid postoperative complications, there must be enough space between the lateral fixtures and mental foraminae. The "zone safety" counts for $2 \mathrm{~mm}$ for minimum (19). Alternatively one may distalize the nerves laterally at first (14) and then continue with implant placement.

5. Sagittal discrepancy is another reason not to indicate the Novum system. In situation of distoocclusion, the final position of bar and denture would be too distal which may disturb the movement space for a tongue. Contrary to this, too ventrally positioned bar and teeth do not allow normoocclusion. In both skeletal disturbances, we encourage patients to go through conventional implant placement.

6. Preparing the mandibular superior alveolar and basal bone, it is critical to parallel the maxillary plane of occlusion and reduce enough bone to leave vertical space for denture teeth and prosthesis componentry at the patient's predetermined vertical dimension of occlusion. If the planar reduction is misdirected, the resultant tilt of the bar structures may create reduced or insufficient room for arranging teeth and processing. This most commonly occurs as a result of the loss of orientation to the occlusal plane as bone reduction proceeds with the mouth open. The osteoplasty is performed not only in the frontal region to meet the sufficient width of the crest, but also in lateral zones where must be enough space under the upper bar for good oral hygiene.

7. The position of the first medial implant of the Novum system defines the final settling of the bar and more less the denture, as well (Fig. 7). Hence, already the location as well as angulation of the first pilot bur is crucial for the success and any displacement is fatal. Performing the conventional interforaminal implantation, it is possible to overcome the mispreparation shifting other implants or using different types of abutments.

8. All the machined Novum implants must be primary stabile, otherwise we can not use them for immediate loading. If one implant fails, the entire superstructure must be removed and after 3-4 months a reimplantation can be performed. Using hybrid bridge with 6 implants, it is possible to keep the prosthesis even when 1 implant is lost.

9. The final Novum denture is delivered in the same day of the surgery, which is time and money saving. Normally, the denture is easy and quick. However, if final position of the lower bar is tilted, the prosthodontic reconstruction is difficult. The bars are prefabricated and we are able to do only small modification.

10. Pulling the lower lip down, the shining lower bar is visible since the hybrid denture is only on the upper bar of the Novum system. For the patients who desire the advanced cosmetics, it is possible to cover the frontal rim of the lower bar with an acrylic veneer connected to the upper bar (Fig. 8).

11. The immediate final denture on Novum implants is a methylmetacrylate made prosthesis with a bar core 
that serves to a patient the same evening as any other final fixed prosthesis. To be able to chew any food, a silicone plate is localized over the sutured wound to preserve the stitches around fixtures. The immediate denture placed on conventional implants is methylmetacrylate with possible fiber or metal reinforcement and is stabilized on provisional abutments. The final restoration is made on the conventional protocol. Lower part of the final custom-made construction has more physiologically looking trimming (Fig. 9). Similarly, the metal part of the restoration is usually visible.

12. Both the immediately loaded conventional implants and the Novum system are well documented, although the lack of long-term studies is typical for both immediate loading techniques. The resorption of the bone adjacent to the implant collar does not differ in any of the procedure. The reported mean loss of bone supported in Novum fixtures did not overcome $0.2 \mathrm{~mm}$ per year (4). Randow et al. (23) studied the marginal bone loss at immediately loaded two-phase implants; a mean value of the resorption after 18 months was $0.4 \mathrm{~mm}$. Moreover, bone responses to the implants placed and loaded immediately are similar to the responses measured at conventionally (delayed) loaded implants (5).

13. Based on literature review, failures of both techniques are similar. Brånemark Osseointegration Center reported the success rates of $98 \%$, placing 150 Novum fixtures and using them with prosthesis over 3 years (11). Ganeles and co-workers (12) published the study experiencing similar number of fixtures (161) placed in edentulous jaw and immediately loaded with a provisional fixed prosthesis. The final screwed dentures were fixed two months later. All implants showed good primary stability and about one third of the patients had their teeth or a fixed denture in the upper jaw. The success rate over 2 years was $99 \%$.

\section{Conclusions}

With appropriate stabilization and occlusal loading, mandibular implants can be immediately loaded in a complete-arch configuration with no apparent detrimental effect on the rates of osseointegration. The Novum System offers a unique opportunity to create a definitive, immediately loaded fixed prosthesis on the edentulous mandible for patients who meet specific presurgical criteria. Since this system is based on precisely placed implants and prefabricated bar structures, the surgical techniques are critical in obtaining a satisfactory result. The same-day loading of conventional implants in the edentulous lower jaw offers a provisional restoration. The results of this short-term clinical (clinical success rate reaching $97.6 \%$ ) and radiographic study (mean marginal bone loss of $0.4 \mathrm{~mm}$ ) evaluating two ways of immediate rehabilitation of edentulous mandible indicate that the precise surgical and prosthetic protocol allows successful prosthetic rehabilitation of mandibular edentulism and that the provisional or permanent fixed reconstruction can be provided to the patient on the day of fixture surgery.

\section{Acknowledgments}

The authors would like to acknowledge the laboratory technicians who contributed their knowledge, ingenuity, and skills: Martina Kubátová (Stomatologická laboratoř Martina Kubátová, Mladá Boleslav) and Jiři Macho (Macho, Pešl s.r.o., České Budějovice).

\section{References}

1. Adell R, Lekholm U, Rockler B et al. A 15-year study of osseointegrated implants in the treatment of the edentulous jaw. Int J Oral Surg 1981;10:387-416.

2. Bector JP, Eckert SE, Isaksson A, Keller EE. The influence of mandibular dentition on implant failures in bone-grafted edentulous maxillae. Int J Oral Maxillofac Implants 2002;17:69-77.

3. Brånemark P-I, Adell R, Albrektsson T, Lekholm U, Lunqvist S, Roskler B. Osseointegrated titanium fixtures in the treatment of edentulousness. Biomaterials 1983;4:25-8.

4. Brånemark P-I, Engstrand P, Öhrnell L-O, Grondahl K, Nilsson P, Hagberg K Brånemark Novum ${ }^{\circledR}$ : A new concept for rehabilitation of the edentulous mandible. Preliminary results from a prospective clinical follow-up study. Clin Implant Dent Rel Res 1999;1:2-16.

5. Brånemark P-I, Hansson BO, Adell R et al. Osseointegrated implants in the treat ment of the edentulous jaw. Experience from a 10-year period. Scand J Plast Reconstr Surg 1977;11(suppl 16):1-132.

6. Brunski JB, Moccia AF, Pollock SR et al. The influence of functional use of endosseous dental implants on the tissue implant interface: I. Histological aspects. J Dent Res 1979;58:1953-69.

7. Buser D, Mericske-Stern R, Bernard JP et al. Long-term evaluation of non-submerged ITI implants. Part 1: 8-year life table analysis of a prospective multicenter study with 2359 implants. Clin Oral Implants Res 1997;8:161-72.

8. Chiapasco M, Abati S, Romeo E et al. Implant-retained mandibular overdentures with Brånemark system MKII implants: a prospective comparative study between delayed and immediate loading. Int J Oral Maxillofac Implants 2001;16:537-46.

9. Chow J, Hui E, Li D, Liu J. Immediate loading of Brånemark System fixtures in the mandible with a fixed provisional prosthesis. Applied Osseointegration Research 2001;1:30-5

10. Cooper LF, Rahman A, Moriarty NC, Sacco D. Immediate mandibular rehabilitation with endosseous implants: simultaneous extraction, implant placement, and loading. Int J Oral Maxillofac Implants 2002;17:517-25.

11. Engstrand P, Nannmark V, Martensson C, Galens I, Brånemark P-I. Brånemark Novum ${ }^{\circledR}$ : Prosthodontic and dental laboratory procedures for fabrication of a fixed prosthesis on the day of surgery. Int J Prosthodont 2001;14:303-8.

12. Ganeles J, Rosenberg MM, Holt RL, Reichman LH. Immediate loading of implants with fixed restorations in the completely edentulous mandible: report of 27 patients from a private practice. Int J Oral Maxillofac Implants 2001;16:418-26.

13. Henry PJ, Rosenberg I. Single-stage surgery for rehabilitation of the edentulous mandible. Preliminary results. Pract Periodont Aesthetic Dent 1994;6:1-9.

14. Krug J, Kasabah S, Mounajjed, M, Cevallo-Lecaro MD, Šimůnek A. Lateralizace nervus alveolaris inferior v kombinaci s dentální implantací. Kazuistika. Quintessenz 2002;2:47-50

15. Lekholm U, Gunne J, Henry P et al. Survival of the Brånemark implant in the partially edentulous jaw. A 10 -year prospective multi-center study. Int J Oral Maxillofac Implants 1999;2:46-53.

16. Lekholm U, Zarb GA. Patient selection and preparation. In: Brånemark P-I, Zarb GA, Albrektsson T (ed.), Tissue-integrated prostheses: osseointegration in clinical dentistry. Chicago: Quintessence, 1985:199-209.

17. Lekholm U. Patient selection for Brånemark Novum Treatment. Applied Osseointegration Research 2001;1:36-9.

18. Misch EC. Density of Bone: Effect on Surgical Approach and Healing. In: Misch EC. Contemporary Implant Dentistry, St. Louis: Mosby, 1999:371-84.

19. Misch EC. Root form surgery in the edentulous mandible: Stage I Implant Insertion. In: Misch EC. Contemporary Implant Dentistry, St. Louis: Mosby, 1999:347-69.

20. Parel SM, Ruff SL, Triplett RG, Schow R. Bone reduction surgical guide for the Novum implant procedure: Technical note. Int J Oral Maxillofac Implants 2002;17:715-9.

21. Piatelli A, Ruggieri A, Franchi M et al. A histologic and histomorphometric study of bone reactions to unloaded and loaded non-submerged single implants in monkeys: A pilot study. J Oral Implantol 1993;19:314-20. 
22. Pilliar RM, Lee GM, Maniatopoulos C. Observations on the effect of movement on bone ingrowth into porous surfaced implants. Clin Orthop Rel Res 1986; 208:108-13

23. Randow K, Ericsson I, Nilner K. et al.: Immediate functional loading of Brånemark dental implants: An 18-month clinical follow-up study. Clin Oral Implants Res 1999;10:8-15.

24. Schnitman PA, Wohrle PS, Rubenstein JE, DaSilvia JD, Wang N-H. Ten-year results for Brånemark implants immediately loaded with fixed prostheses at implant placement. Int J Oral Maxillofac Implants 1997;12:96-105.

Submitted April 2003.

Accepted September 2003.

MUDr. et MUDr. Jiři Krug, Ph.D.,

D.C.M., private dental office,

Hradební 856, 50002 Hradec Králové,

Czech Republic.

e-mail: jirikrug@hotmail.com 